\title{
173. Remarks on Generalized Rings of Quotients
}

\author{
By Tomoharu AKIBA \\ Department of Education, Kobe University \\ (Comm. by Zyoiti Suetuna, M.J.A., Dec. 12, 1964)
}

Introduction. Let $A$ be an integral domain and let $B$ be an overring of $A$ contained in the quotient field of $A$. Then $B$ is called a generalized ring of quotients of $A$ if $B$ is flat as an $A$-module. It has been shown that generalized rings of quotients have similar properties to those of ordinary rings of quotients (see [2] and [6]). In $\S 1$ of this paper, we first generalize the results to the case where $A$ is not necessarily an integral domain. Some of the proofs are adaptions of those of [6], but, in order to make this paper self-contained, we repeat them again. In $\S 2$, we give a counter example to the following conjecture of Richman in [6].

Let $A$ be an integral domain and let $B$ be a generalized ring of quotients of $A$ not equal to $A$. Then there exists an $x / y$ in $B$ which is not in $A$, such that $(x, y) A$ is invertible.

The author wish to express his heartful thanks to Prof. M. Nagata for kind advices and for valuable suggestions.

$\S 1$. First of all, we list some well-known properties of flatness as lemmas without proofs (cf. [1], [3], [4]). Rings will mean always commutative rings with units.

Lemma 1. Let $R$ and $R^{\prime}$ be rings such that $R^{\prime}$ is an $R$-module. Then $R^{\prime}$ is $R$-flat if and only if the following condition is satisfied:

If $\left(y_{i}\right)$ and $\left(c_{i}\right)$ are finite subsets of $R^{\prime}$ and $R$ respectively, such that $\sum_{i} c_{i} y_{i}=0$, then there exist a finite subset $\left(x_{j}\right)$ of $R^{\prime}$ and a finite subset $\left(b_{i j}\right)$ of $R$ for which we have $\sum_{i} b_{i j} c_{i}=0$ for each $j$, and $y_{i}=$ $\sum_{j} b_{i j} x_{j}$ for each $i$.

Lemma 2. Let $R$ and $R^{\prime}$ be as above and assume that $R^{\prime}$ is $R$ flat. Let $\mathfrak{a}_{1}, \cdots, \mathfrak{a}_{r}$ be ideals of $R$. Then we have $\left(\bigcap_{i} \mathfrak{a}_{i}\right) R^{\prime}=\bigcap_{i} \mathfrak{a}_{i} R^{\prime}$.

Let $A$ be a ring. In this section, we shall denote by $B$ an overring of $A$ contained in the total quotient ring of $A$.

Theorem 1. The following three conditions are equivalent to each other:

(1) $B$ is A-flat.

(2) For any element $b$ of $B$, we have $(A: b) B=B$, where $(A: b)$ denotes the set of elements $a$ of $A$ such that $a b \in A$. (It is evident that $(A: b)$ is an ideal of $A$.) 
(3) For every prime ideal $\mathfrak{p}^{\prime}$ of $B$, the canonical homomorphism $\varphi_{\mathfrak{p}^{\prime}}$ from $A_{\mathfrak{p}^{\prime} \cap \mathbf{A}}$ in $B_{\mathfrak{p}^{\prime}}$ is bijective. (Cf. Theorems 1 and 2 in [6].)

Proof. Equivalence between (1) and (2): Assume that $B$ is $A$ flat. Let $b=x / y(x, y \in A ; y$ is not a zero divisor) be an element of $B$. Then $y(x / y)-x .1=0$. By Lemma 1 , there exist a finite subset $\left(b_{j}\right)$ of $B$ and a finite subset $\left(a_{1 j}, a_{2 j}\right)$ of $A$ such that $\left(1^{\prime}\right) a_{1 j} y-a_{2 j} x=0$ for each $j,\left(2^{\prime}\right) x / y=\sum_{j} a_{1 j} b_{j}$, and $\left(3^{\prime}\right) 1=\sum_{j} a_{2 j} b_{j}$. From $\left(1^{\prime}\right)$, it follows that $a_{2 j}$ is in $(A: b)$ for every $j$, and $\left(3^{\prime}\right)$ asserts that $(A: b) B=B$. Thus (1) implies (2).*) Assume, conversely, that (2) is valid. Let $\left(y_{i}\right)$ and $\left(c_{i}\right)$ be finite subsets of $B$ and $A$ respectively, such that $\sum_{i} y_{i} c_{i}=0$. Since $\left(A: y_{i}\right) B=B$ for each $i$ by the condition (2), we have $\left(\bigcap_{i}\left(A: y_{i}\right)\right) B \supseteqq \prod_{i}\left(A: y_{i}\right) B \supseteqq B$, and $\left(\bigcap_{i}\left(A: y_{i}\right)\right) B=B$. So there are finite subsets $\left(a_{j}\right)$ and $\left(x_{j}\right)$ of $\cap\left(A: y_{i}\right)$ and $B$ respectively, for which we have $\sum_{j} a_{j} x_{j}=1$. Then $b_{i j}=y_{j} a_{j}$ is in $A$ for each $i$ and $j, y_{i}=$ $\sum_{j} y_{i} a_{j} x_{j}=\sum_{j}^{j} b_{i j} x_{j}$ for every $i$, and $0=\sum_{i} c_{i} y_{i} a_{j}=\sum_{i} c_{i} b_{i j}$ for each $j$. By Lemma 1 , this shows that $B$ is $A$-flat. Hence (2) implies (1).

Equivalence between (2) and (3): assume that (2) is true. Let $\mathfrak{p}^{\prime}$ be an arbitrary prime ideal of $B$ and set $\mathfrak{p}=\mathfrak{p}^{\prime} \cap A$. If $\varphi_{\mathfrak{p}^{\prime}}(a / s)=0$ for an $a / s$ of $A_{\mathfrak{p}}(a \in A, s \in A-\mathfrak{p})$, then we have $a s^{\prime}=0$ with an $s^{\prime}$ in $B-\mathfrak{p}^{\prime}$. By the condition (2), $\left(A: s^{\prime}\right) B=B$ and then $\left(A: s^{\prime}\right) \nsubseteq \mathfrak{p}$. Hence there is an element $t$ in $\left(A: s^{\prime}\right)$ such that $t \notin \mathfrak{p}$. Then $a s^{\prime} t=0$ with $s^{\prime} t \in A-\mathfrak{p}$, which shows that $a / s=0$ in $A_{\mathfrak{p}}$, whence $\varphi_{\mathfrak{p}^{\prime}}$ is injective. Next, let $b / s^{\prime}$ be an arbitrary element of $B_{\mathfrak{p}^{\prime}}\left(b \in B, s^{\prime} \in B-\mathfrak{p}^{\prime}\right)$. Since $(A: b) B=B$ and $\left(A: s^{\prime}\right) B=B$, we have $\left((A: b) \cap\left(A: s^{\prime}\right)\right) B=B$ and so $(A: b) \cap\left(A: s^{\prime}\right) \nsubseteq \mathfrak{p}$. Then there is an element $s$ in $(A: b) \cap\left(A: s^{\prime}\right)$ which is not in $\mathfrak{p}$. For the $s$, we have $s s^{\prime} \in A-\mathfrak{p}$ and $b s \in A$, so $b s / s s^{\prime}$ may be considered as an element of $A_{\mathfrak{p}}$. It is obvious that $\varphi_{\mathfrak{p}^{\prime}}\left(b s / s s^{\prime}\right)=$ $b / s^{\prime}$, which shows that $\varphi_{\mathfrak{p}^{\prime}}$ is surjective. Thus (2) implies (3). Conversely, assume that (3) is satisfied, and suppose that there is a $b \in B$ such that $(A: b) B \neq B$. Then there is a prime ideal $\mathfrak{p}^{\prime}$ of $B$ containing $(A: b) B$, and so we have $\mathfrak{p} \supseteqq(A: b)$ for $\mathfrak{p}=\mathfrak{p}^{\prime} \cap A$. Since $\varphi_{\mathfrak{p}^{\prime}}$ is surjective by the condition (3), we can take $a \in A$ and $s \in A-\mathfrak{p}$ so that $\varphi_{\mathfrak{p}^{\prime}}(a / s)=b / 1$, which implies that $(a-b s) s^{\prime}=0$ for some $s^{\prime} \in B-\mathfrak{p}^{\prime}$. From the assumption that $B$ is contained in the total quotient ring of $A$, it follows that there is a $t \in(A: b)$ which is not a zero divisor in $A$ and so in $B$. Then $(a t-b s t) s^{\prime}=0$, which shows that $\varphi_{\mathfrak{p}^{\prime}}(a t / 1)=$ $\varphi_{p^{\prime}}(b s t / 1)$. So there is an $r \in A-\mathfrak{p}$ such that $(a t-b s t) r=0$ because $\varphi_{p^{\prime}}$ is injective by (3). From this we have $a r-b s r=0$, since $t$ is not a zero divisor. Hence we have $s r \in A-\mathfrak{p}$ and $s r \in(A: b)$, which is a contradiction because $\mathfrak{p} \supseteqq(A: b)$. Thus we have $(A: b) B=B$ for all

*) This part of the proof is the same as that of [6]. 
$b$ of $B$, and (3) implies (2).

Adapting [6], an overring $B$ of a ring $A$ contained in the total quotient ring of $A$ is said to be a generalized ring of quotients of $A$ if $B$ is $A$-flat.

Corollary 1. Let $B$ be a generalized ring of quotients of $A$. Then for any overring $C$ of $A$ contained in $B, B$ is a generalized ring of quotients of $C$. (Cf. Lemma 2 in [6].)

The proof is straightforward and we omit it.

Corollary 2. If a generalized ring of quotients $B$ of $A$ is integral over $A$, then $A=B$. (Cf. Proposition 2 in [6].)

The proof follows directly from Theorem 1 and the fact that if $B$ is integral over $A$, then the extended ideal of a proper ideal of $A$ to $B$ is again proper.

Corollary 3. Let $B$ be a generalized ring of quotients of $A$, and let $A^{*}$ and $B^{*}$ be integral closures of $A$ and $B$ respectively, in the total quotient ring of $A$. Then $B^{*}=B\left[A^{*}\right]$, and $B^{*}$ is a generalized ring of quotients of $A^{*}$. In particular, if $A$ is integrally closed in its total quotient ring, then $B$ is also integrally closed in its total quotient ring. (Cf. Proposition 1 and Corollary in [6].)

Proof. Let $b^{*}$ be an element of $B^{*}$, then $b^{* n}+b_{1} b^{* n-1}+\cdots+b_{n}=0$ with $b_{i} \in B$. From Theorem 1 , it follows that $\left(A: b_{i}\right) B=B$ for every $i$ and so $\left(\bigcap_{i}\left(A: b_{i}\right)\right) B=B$. Then there are finite subsets $\left(a_{j}\right)$ and $\left(c_{j}\right)$ of $\cap\left(A: b_{i}\right)$ and $B$ respectively, such that $\sum_{j} a_{j} c_{j}=1$. Since $a_{j} b^{*}$ is in $A^{*}$ for every $j$, we have $b^{*}=\sum_{j} a_{j} c_{j} b^{*} \in B\left[\stackrel{j}{A}^{*}\right]$, which shows that $B^{*} \subseteq B\left[A^{*}\right]$. The converse inclusion being obvious, $B^{*}=B\left[A^{*}\right]$. Since, under the above notations, $\left(A^{*}: b^{*}\right) \supseteqq \bigcap_{i}\left(A: b_{i}\right)$, we see that $B^{*}$ is a generalized ring of quotients of $A^{*}$ by Theorem 1 and the definition. The last assertion is trivial. Then:

Theorem 2. Let $B$ be a generalized ring of quotients of $A$.

(1) For any ideal $\mathfrak{b}$ of $B$, we have $(\mathfrak{b} \cap A) B=\mathfrak{b}$. In particular, prime ideals of $B$ are generated by prime ideals of $A$.

(2) Let $\mathfrak{q}$ be a primary ideal of $A$ belonging to a prime ideal $\mathfrak{p}$ and such that $\mathfrak{q} B \neq B$. Then $\mathfrak{p} B \neq B, \mathfrak{p} B$ is a prime ideal, $\mathfrak{q} B$ is primary to $\mathfrak{p} B, \mathfrak{p} B \cap A=\mathfrak{p}$, and $\mathfrak{q} B \cap A=\mathfrak{q}$. (Cf. Theorem 3 in [6].)

Proof. Let $b$ be an element of $\mathfrak{b}$. Since $(A: b) B=B$ by Theorem 1 , there are finite subsets $\left(a_{i}\right)$ and $\left(b_{i}\right)$ of $(A: b)$ and $B$ respectively, such that $\sum_{i} a_{i} b_{i}=1$. Then $a_{i} b \in \mathfrak{b} \cap A$ for every $i$, and $b=\sum_{i} a_{i} b_{i} b$ is in $(A: b) B$, which shows that $(\mathfrak{b} \cap A) B \supseteqq \mathfrak{b}$. Since the converse inclusion is clear, we have $(\mathfrak{b} \cap A) B=\mathfrak{b}$. Thus (1) is proved. The first assertion in (2) is trivial. Next, we shall prove the other assertions 
in (2). If $q \in \mathfrak{q} B \cap A$, then $q=\sum_{i} q_{i} b_{i}$ with $q_{i} \in \mathfrak{q}, b_{i} \in B$. Since $\left(A: b_{i}\right) B=$ $B$ for each $i$ by Theorem 1 , we have $\left(\bigcap_{i}\left(A: b_{i}\right)\right) B=B$. From $\mathfrak{p} B \neq B$, it follows that $\mathfrak{p} \bigcap_{i}\left(A: b_{i}\right)$, hence there is an $a$ in $\bigcap_{i}\left(A: b_{i}\right)$ which is not in $\mathfrak{p}$. Then $a q \in \mathfrak{q}$ and, since $a \notin \mathfrak{p}$, we have $q \in \mathfrak{q}$. This shows that $\mathfrak{q} B \cap A \subseteq q$. On the other hand, that $\mathfrak{q} B \cap A \supseteqq \mathfrak{q}$ is clear and we have $\mathfrak{q} B \cap A=\mathfrak{q}$. As a particular case where $\mathfrak{q}=\mathfrak{p}$, we have $\mathfrak{p} B \cap A=\mathfrak{p}$. Now, let $b$ and $b^{\prime}$ be elements of $B$ such that $b b^{\prime} \in \mathfrak{q} B$ and $b^{\prime} \notin \mathfrak{q} B$. Then there is an $a^{\prime} \in\left(A: b^{\prime}\right)$ such that $a^{\prime} b^{\prime} \notin \mathfrak{q}$. In fact, otherwise, we would have $b^{\prime} \in b^{\prime}\left(A: b^{\prime}\right) B \cong \mathfrak{q} B$ because $\left(A: b^{\prime}\right) B=B$, which is a contradiction. Furthermore, since $(A: b) B=B$ there are $a_{1}, \cdots, a_{r}$ in $(A: b)$ such that $\mathfrak{a} B=B$ where $\mathfrak{a}=\left(a_{1}, \cdots, a_{r}\right) A$. Then it is obvious that for any positive integer $n, \mathfrak{a}^{n} B=B$. On the other hand, we have $a_{i} a^{\prime} b b^{\prime}=a_{i} b a^{\prime} b^{\prime} \in \mathfrak{q} B \cap A=\mathfrak{q}$ for $i=1, \cdots, r$. Since $a^{\prime} b^{\prime} \notin \mathfrak{q}$ and since $\mathfrak{q}$ is a primary ideal, it follows that there is a positive integer $n_{i}$ such that $\left(a_{i} b\right)^{n_{i}} \in \mathfrak{q}(i=1, \cdots, r)$. Then, taking a positive integer $n$ to be $n \geqq \max \left\{r n_{i}\right\}$, we have $b^{n} \in b^{n} a^{n} B \leqq q B$ as can be easily seen, which shows that $q B$ is a primary ideal. Applying this to the case where $\mathfrak{q}=\mathfrak{p}$, we see that $\mathfrak{p} B$ is a prime ideal because in that case $n$ can be taken to be 1 . Any element of $\mathfrak{p}$ being nilpotent modulo $\mathfrak{q}$, elements of $\mathfrak{p} B$ are also nilpotent modulo $\mathfrak{q} B$, whence $\mathfrak{q} B$ belongs to $\mathfrak{p} B$. Thus the proof of Theorem 2 is complete.

Corollary 1. If $A$ is Noetherian, then any generalized ring of quotients of $A$ is Noetherian. (Cf. Corollary of Theorem 3 in [6].)

This follows immediately from the above theorem and the wellknown theorem of Cohen (see (3.4) of Chap. 1 in [4]).

The following corollary is an immediate consequence of Theorem 2 and Lemma 2.

Corollary 2. Let $B$ be a generalized ring of quotients of $A$ and let $\mathfrak{q}_{1}, \cdots, \mathfrak{q}_{r}$ be primary ideals of $A$ such that $\mathfrak{q}_{i} B \neq B$ for every $i$. Set $\mathfrak{a}=\mathfrak{q}_{1} \cap \cdots \cap \mathfrak{q}_{r}$. Then $\mathfrak{a} B=\mathfrak{q}_{1} B \cap \cdots \cap \mathfrak{q}_{r} B$ and $\mathfrak{a} B \cap A=\mathfrak{a}$. (Cf. Theorem 3 in [6].)

$\S 2$. We shall give a counter example to the conjecture of Richman (see Introduction) in the case where $A$ is a local integral domain. In that case, condition that $(x, y) A$ is invertible implies that $(x, y) A$ is principal, say $(x, y) A=z A$. Then $(x / z, y / z) A=A$. Since $A$ is local, one of $x / z$ and $y / z$ is a unit, whence $(x, y) A=x A$ or $(x, y) A=y A$. But $x / y$ is not in $A$ by our assumption, so we have $(x, y) A=x A$. Then $y / x$ is in $A$ and is invertible in $B$.

Therefore, for our purpose, it is enough to construct a local integral domain $A$ and a generalized ring of quotients $B$ of $A$ such that $B \neq A$ and no non-unit of $A$ is invertible in $B$. In the following, the notations will be as in [5]. 
Let $C$ be a non-singular plane cubic curve defined over a field $k_{0}$ and let $P$ be a generic point of $C$ over $k_{0}$, and let $k$ be a field containing $k_{0}(P)$. Then the homogeneous coordinate ring $R_{0}=k[x, y, z]$ of $C$ over $k$ is normal. Let $R=k[x, y, z]_{(x, y, z)}$ and $R^{\prime}=\cup_{n} \mathfrak{p}^{-n}$ (p-transform of $R$ in the sense of [5]), where $\mathfrak{p}$ is the homogeneous prime ideal of $R$ corresponding to $P$.

We shall show that $A=R$ and $B=R^{\prime}$ give the required example.*)

First, we shall prove that no non-unit of $R$ is invertible in $R^{\prime}$. Suppose that there is an $f$ of $R$ such that $f$ is non-unit in $R$ and $f^{-1} \in R^{\prime}$. Then $\mathfrak{p}^{n} \cong f R$ for some $n$ and the normality of $R$ implies that $f R$ is primary to $\mathfrak{p}$. Therefore $f R=\mathfrak{p}^{(m)}(m$-th symbolic power of $\mathfrak{p})$ for a suitable $m$. Since $\mathfrak{p}^{(m)}=\mathfrak{p}_{0}^{(m)} R\left(\mathfrak{p}_{0}=\mathfrak{p} \cap R_{0}\right)$ and since $\mathfrak{p}_{0}^{(m)}$ is homogeneous, we may assume that $f$ is a homogeneous element of $R_{0}$. Then $f R_{0}=\mathfrak{p}_{0}^{(m)}$, and this shows that the intersection of the hypersurface $f=0$ with $C$ is $m P$, which is a contradiction because $P$ is a generic point and $C$ is of positive genus.

Next, we shall prove that $R^{\prime}$ is $R$-flat.

Lemma. Let $\mathfrak{O}$ be an integral domain and let $\mathfrak{a}$ be an ideal of $\mathfrak{D}$. Set $\mathfrak{D}^{\prime}=\cup \mathfrak{a}^{-n}$. Then there exists a one to one correspondence between prime ideals $\mathfrak{q}^{\prime}$ of $\mathfrak{S}^{\prime}$ and prime ideals $\mathfrak{q}$ of $\mathfrak{D}$ except those containing $\mathfrak{a} \mathfrak{D}^{\prime}$ and a respectively, in such a way that $\mathfrak{q}^{\prime}$ corresponds to $\mathfrak{q}=\mathfrak{q}^{\prime} \cap \mathcal{O}$. In the case we have $\mathfrak{O}_{\mathfrak{q}^{\prime}}^{\prime}=\mathfrak{O}_{\mathfrak{q}}$. (Cf. Lemma 3 of $\S 1$ in [5].)

By the above lemma and Theorem 1, it is sufficient to prove that $\mathfrak{p} R^{\prime}=R^{\prime}$. Let $R_{0}^{\prime}=\bigcup_{n} \mathfrak{p}_{0}^{-n}$. Since $P$ is rational over $k$, $\mathfrak{p}_{0}$ can be generated by linear forms. If $t$ is a linear form contained in $\mathfrak{p}_{0}$, then any element of $R_{0}^{\prime}$ is of the form $q / t^{n}$ with $q \in t^{n} R_{0}: \mathfrak{p}_{0}^{n}$ for a suitable $n$ (see [5]). Assume that $q$ is homogeneous of degree $d$. For a homogeneous element $g$ of $R_{0}$, we denote by $D_{g}$ the divisor of $C$ which is cut out by the hypersurface $g=0$. Then $q \in t^{n} R_{0}: \mathfrak{p}_{0}^{n}$ if and only if $D_{q}+n P>n D_{t}$. Since $C$ is a non-singular plane curve, the system of hypersurface sections of a given degree is complete. Furthermore, since the genus of $C$ is 1 , for any divisor $D$ of degree greater than 1 , the complete system $|D|$ has no fixed points. Then it follows that, taking $n$ and $d$ so that $3 d-2 n>2$ and $n>d$, we can find two homogeneous forms $q_{1}$ and $q_{2}$ of degree $d$ in $t^{n} R_{0}: \mathfrak{p}_{0}^{n}$ such that $D_{q_{1}}-n D_{t}$ and $D_{q_{2}}-n D_{t}$ have no common points except $P$. Taking two linear forms $h_{1}$ and $h_{2}$ belonging to $\mathfrak{p}_{0}$ so that $D_{q_{1} h_{1}}-n D_{t}$ and $D_{q_{2} h_{2}}-n D_{t}$ have no common points except $P$, we set $a_{1}=q_{1} h_{1}^{n-a} / t^{n}$ and $a_{2}=q_{2} h_{2}^{n-a} / t^{n}$.

On the other hand, $C-\{P\}$ is an affine curve and so we denote its affine ring by $R^{*}$. Then $a_{1}$ and $a_{2}$ are contained in $R^{*}$ and

*) This example was obtained following a suggestion made by Prof. Nagata 
$a_{1} R^{*}+a_{2} R^{*}=R^{*}$ because $a_{1}$ and $a_{2}$ have no common zeros. Obviously, $R^{\prime} \supseteqq R^{*}$ and, since $q_{i} / t^{n} \in R^{\prime}$ and $h_{i} \in \mathfrak{p}$, we have $a_{i} \in \mathfrak{p} R^{\prime}(i=1,2)$. Then the relation $a_{1} R^{*}+a_{2} R^{*}=R^{*}$ implies that $\mathfrak{p} R^{\prime}=R^{\prime}$, as we wanted.

\section{References}

[1] N. Bourbaki: Algèbre Commutative. Hermann, Paris (1961).

[2] L. Budach: Über eine Charakterisierung der Grellschen Schemata. Math. Nachr., 27, 5-6 (1964).

[3] M. D. Lazard: Sur les modules plats. C. R. Acad. Sci. Paris, 258 (1964).

[4] M. Nagata: Local Rings. Interscience, New York (1962).

[5] — : A treatise on 14th problem of Hilbert. Mem. Coll. Sci.U niv. Kyoto, 30 (1) (1956); Corrections, ibid, 30 (3) (1957).

[6] F. Richman: Generalized quotient rings, forthcoming.

[7] R. Walker: Algebraic Curves. Princeton University Press (1950). 